\title{
CERCAS VIVAS Y SU IMPORTANCIA AMBIENTAL EN LA CONSERVACIÓN DE AVIFAUNA NATIVA
}

\section{LIVE FENCES AND ITS ENVIRONMENTAL IMPORTANCE IN THE CONSERVATION OF NATIVE AVIFAUNA}

\author{
DE LA OSSA-LACAYO, ALEJANDRO ${ }^{*}$ Ecólogo. \\ ${ }^{1}$ Universidad de Sucre - SUE Caribe, Maestría en Ciencias Ambientales, Grupo \\ de Investigación en Biodiversidad Tropical, Colombia. \\ *Correspondencia: alejandrodelaossa@yahoo.com
}

Recibido: 19-02-2013; Aceptado: 15-05-2013.

\section{Resumen}

El presente trabajo hace un análisis de la literatura científica de mayor importancia que a nivel de zonas tropicales de América latina se tiene para los sistemas agrosilvopastoriles, enfatizando en la valoración ambiental de las cercas vivas, que como elementos importante de este tipo de paisaje agrícola tienen en la conservación de avifauna nativa. Igualmente hace referencia al valor productivo de las cercas vivas y su papel como sistema que permite el flujo de fauna silvestre y conecta remanentes de bosques y parches, así como elementos arbóreos aislados en una matriz altamente fragmentada.

Palabras clave: cercas vivas, avifauna, importancia ambiental, paisaje agrícola.

\begin{abstract}
The present work makes an analysis of the most important scientific literature available for agri-silvopastoral systems in tropical zones of Latin America, focusing on the environmental valuation of live fences, which as important element in this type of agricultural landscape has on the conservation of native avifauna. Equally it refers to the productive value of the live fences and its paper as system that allows the flow of wildlife and connects forests remnants and patches, as well as arboreal elements isolated in a highly fragmented matrix.
\end{abstract}

Key words: live fences, avifauna, environmental importance, agricultural landscape.

\section{Introducción}

Desde los años 50, con mayor énfasis, gran parte del área boscosa original en Latinoamérica se ha deforestado para promover la ganadería extensiva (SERRAO y TOLEDO, 1990; KAIMOWITZ, 2001; KAIMOWITZ y ANGELSEN, 2008). Como lo señalan LANG et al. (2003) para el trópico americano, y en muchos países como Colombia, zonas bajas fueron convertidas en grandes 
áreas de pasturas para la producción ganadera, dando como resultado un paisaje de pequeños fragmentos de bosque dentro de una matriz agropecuaria, la cual a veces solo posee de su original vegetación árboles dispersos y cercas vivas, que se constituyen en valiosos refugios de fauna silvestre regional (LOISELLE y BLAKE, 1992).

Como lo asevera SOMARRIBA (1990) los sistemas silvopastoriles son una modalidad de los sistemas agroforestales, definidos como una forma de cultivo múltiple en la que se deben cumplir tres condiciones fundamentales: 1) que existan, al menos, dos especies de plantas que interactúen biológicamente, 2) al menos uno de los componentes debe ser una leñosa perenne, y 3) al menos uno de los componentes debe ser una planta manejada con fines agrícolas, incluyendo pastos, lo que permite, según la definición y la estructura incluir, entre otros, los potreros tradicionales de muchas de las fincas ganaderas de la región Caribe colombiana en relación con su estructura florística, manejo del componente vegetal, y promoción y mantenimiento de cercas vivas como sistemas agroforestales.

Los sistemas silvopastoriles son sin duda una alternativa de manejo sostenible, tal como lo plantean diferentes autores (HARVEY y HABER, 1999; BEER et al., 2003; HARVEY et al., 2005, 2008). Poseen importante potencial para proveer hábitats y recursos e incrementar la conectividad del paisaje agropecuario. Además, pueden permitir el movimiento animal a través de áreas agropecuarias y al mismo tiempo ayudar a incrementar la productividad y diversificación de productos en las fincas ganaderas (TOBAR-LÓPEZ e IBRAHIM, 2008).

Las cercas vivas se definen como elementos lineales divisorios arbolados que separan áreas de pasturas, áreas de cultivos y algunos parches de bosques (HARVEY et al., 2005). Las cercas vivas, estructuras dominantes en paisajes agrícolas, son denominadas también rompevientos, bordes 0 terrazas, dependiendo de su estructura y función (ZURIA y GATES, 2006).

Las cercas vivas son elementos conspicuos de los paisajes agrícolas y parte importante de los sistemas silvopastoriles. Sin embargo, existe poca información publicada acerca de su abundancia, distribución y función (HARVEY et al., 2003). La información básica de tipo descriptivo es relativamente reciente, aun cuando su uso es generalizado desde hace más de un siglo (LAGEMANN y HEUVELDOP, 1983); puntualmente se ha estudiado respecto de las cercas vivas: la producción de forraje (BERINGER y SALAS, 2003), el establecimiento (SOMARRIBA, 1995), tasas de crecimiento (BEER, 1987) y capacidad de enraizamiento (LOZANO, 1962). No obstante, de manera amplia la importancia ambiental de las cercas vivas como potenciales hábitat y como corredores biológicos remanentes no han sido profusamente estudiada, 
menos aún se ha dilucidado su importancia en la conectividad entre remanentes boscosos y áreas ganaderas transformadas, salvo algunos trabajos llevados a cabo en México y en Costa Rica (ESTRADA et al., 1993; ESTRADA et al., 2000; ESTRADA y COATES-ESTRADA, 2001; HARVEY et al., 2003).

ENRÍQUEZ et al. (2009) anotan que para la conservación de la biodiversidad en paisajes fragmentados o áreas productivas son de especial importancia los elementos arbóreos que éstas poseen, ya que estos elementos crean una variedad de hábitats que no están presentes en lugares manejados intensivamente por el hombre. Igualmente, puntualizan que los sistemas silvopastoriles combinan el manejo de árboles y pasturas de varios tipos con la producción ganadera, y al integrar árboles dentro de potreros estos sistemas pueden cumplir diferentes funciones y proveer múltiples servicios. Entre otros, ayudan a incrementar la productividad de la finca mientras protegen los recursos naturales, favorecen la diversidad y almacenan buena cantidad de carbono, mitigando el efecto invernadero, lo cual concuerda con los trabajos de HARVEY (2001), CORDERO y BOSHIER (2003), IBRAHIM et al. (2006).

\section{Generalidades}

La transformación y fragmentación de los bosques representan las principales causas de la pérdida de diversidad biológica a nivel global (GALLEGO, 2002; MEYERS et al., 2000; SILVA-MATOS et al., 2005). Como consecuencia de la deforestación y fragmentación de los bosques, hoy en día muchas regiones del planeta han sido transformadas a agropaisajes. Estos agropaisajes generalmente son dominados por una matriz de potreros o cultivos anuales, pero aún retienen alguna cobertura arbórea dispersa en forma de pequeños parches remanentes de bosques, franjas angostas de bosques ribereños (riparios) y árboles dispersos. A veces estos paisajes también incluyen cercas vivas que han sido establecidas por los productores para dividir potreros 0 campos agrícolas y evitar el paso de animales (HARVEY et al., 2003). Enfatizando, según SÁNCHEZ-MERLOS et al. (2005), existe poca información sobre la vegetación que mantienen y de las especies presentes. Tampoco existe información sobre las diferencias en la diversidad y composición de la vegetación presente en los diferentes tipos de cobertura arbórea presentes en zonas de potreros y menos aún para el Caribe colombiano.

Se ha evidenciado que en paisajes altamente fragmentados y dominados por potreros aún se puede mantener una diversidad florística importante (SÁNCHEZ-MERLOS et al., 2005). No es posible conocer a ciencia cierta qué porcentaje retienen estos paisajes de la flora original debido a la ausencia de datos florísticos sobre los bosques originales, pero es probable que muchas especies disminuyan su abundancia o hayan sido eliminadas debido a la alta 
deforestación del paisaje y la actividad ganadera (SÁNCHEZ-MERLOS et al., 2005).

Se tiene por lo general una flora asociada a paisajes ganaderos dominada por especies generalistas de áreas abiertas o perturbadas. La dominancia de estas especies se debe en parte a su alta capacidad de regeneración en áreas degradadas y a su dispersión activa por el ganado (ESQUIVEL et al., 2003), y en parte a la costumbre de los productores de dejar estas especies arbóreas en sus fincas para proveer productos y servicios (MARTíNEZ, 2004; SÁNCHEZMERLOS et al., 2005). La presencia natural de las especies arbóreas que hoy dominan potreros y cercas vivas parecen haber tenido un importante impacto sobre la vegetación actual, ya que la mayoría de las especies comunes en estos paisajes son las mismas que los productores seleccionan y retienen cuando establecen o manejan sus potreros (MARTíNEZ, 2004; SÁNCHEZMERLOS et al., 2005).

Aunque el paisaje ganadero, evidentemente está dominado por especies generalistas, mantiene un importante valor para la conservación, ya que al presentar una mezcla interesante de especies arbóreas, retiene algunas especies típicas del bosque original y puede llegar a albergar especies amenazadas (SÁNCHEZ-MERLOS et al., 2005), incluso puede poseer una flora transicional del bosque original (SALAS, 1993).

Se tienen registros de diferencias marcadas en la diversidad, riqueza, abundancia, y estructura de la vegetación entre los hábitats de una región ganadera. Los bosques ribereños fragmentados pueden poseer mayor riqueza y diversidad de especies que los demás hábitat, debido a condiciones ecológicas favorables como humedad, bajas temperaturas y sombra (PITHER y KELLMAN, 2002; SÁNCHEZ-MERLOS et al., 2005). Las cercas vivas pueden presentar mayores densidades arbóreas y mayores áreas básales, comparativamente, dado que los productores siembran los árboles a muy altas densidades y les dan poco manejo, dejando que los árboles alcancen el estado adulto (HARVEY et al., 2003).

De los paisajes ganaderos de bosque seco tropical, las formaciones ribereñas y secundarias son hábitats con mayor valor para la conservación. Estos hábitat tienen una mayor riqueza y diversidad florística, mantienen mayor complejidad estructural que los demás hábitat circundantes, conservan más especies del bosque nativo, poseen las mayores densidades de árboles pequeños, que se relacionan con una activa regeneración (SÁNCHEZ-MERLOS et al., 2005).

Las cercas vivas y potreros con diferentes densidades arbóreas, comparativamente con los remanentes boscosos secundarios y de ribera, podrían ser de menor valor para la conservación florística, pero pueden retener 
especies con valor maderable, frutal y combustible, también pueden ser importantes fuentes de productos y merecen ser considerados como valiosos sistemas en la conservación (MEDINA et al., 2004; VILCHEZ et al., 2004; SÁNCHEZ-MERLOS et al., 2005). Estos hábitats podrían ser importantes para la vida silvestre dentro de las áreas pecuarias. Además, la presencia de los árboles en las cercas vivas, ayudan a mantener la conectividad física del paisaje y servir como corredores o conectores a parches de bosques aislados probablemente ayudando a algunas especies de animales a movilizarse a través de la matriz agropecuaria (HARVEY y HABER, 1999; HARVEY et al., 2003) e incrementando el flujo genético necesario para el mantenimiento de una alta variabilidad (HASKELL et al., 2001; HILTY y MERENLENDER, 2004; HARVEY et al., 2008).

Existen planteamientos que en términos generales, para el trópico, analizan la crisis y las externalidades negativas de la ganadería bovina con el uso de la tierra, la deforestación, el empleo rural y la biodiversidad, y proponen el desarrollo de diversas alternativas que permitan la transformación de la ganadería actual en un sistema sostenible, menos incompatible con la diversidad biológica y más apropiada para lograr el bienestar humano, dejando en claro que a conservación de zonas boscosas y la utilización de los árboles y los arbustos en los sistemas ganaderos son un buen enfoque en tal sentido (GUTTERIDGE, 1990; SHELTON et al., 1991; FEBLES et al., 1995; PALMA y FLORES, 1997; PALMA, 1998; MURGUEITIO y CALLE, 1998; KU et al., 1998; BOTERO y RUSSO, 1998; RUIZ y FEBLES, 1999; IBRAHIM et al., 2003; PALMA, 2005).

Las cercas vivas constituyen un sistema tradicional en el manejo de fincas tropicales a la vez muy conocido por los productores, pero se necesita más información acerca de las especies arbóreas o arbustivas que se usan como forraje, lo cual podría ofrecer a nivel productivo la disponibilidad de una mayor cantidad de alimento por hectárea, optimizando la disponibilidad de comida animal durante las épocas ambientalmente críticas (PALMA, 2005).

PALMA (2005) señala que en México es común observar en los potreros la presencia de cercas vivas; entre las especies observadas se indican: el papelillo o cuajiote (Bursera simaruba), el vainillo (Senna atomaria), el ciruelo (Spondias mombin), la guácima (Guazuma ulmifolia), el guamúchil (Pithecellobium dulce), cuastecomate (Crescentia alata), asmol (Zizipus mexicana), brasil (Haematoxylon brasiletto), cacanahual (Gliricidia sepium), colorín (Erythrina americana), entre otras. DENIZ (2003) indica, para México también, la plantación en cercas vivas de coral (Caesealpinia platyloba), especie cuyo éxito se debe a la baja gustosidad por los rumiantes y al reconocimiento de los productores dado su alto valor como poste, lo cual es 
otro tipo de enfoque para valorar la importancia puntual de una especie arbórea en las cercas vivas.

\section{Conservación}

La configuración espacial de un paisaje agrosilvopastoril está representado por todos sus elementos naturales o antrópicos, entre ellos las cercas vivas, constituyen una característica clave para asociar las importantes repercusiones que el sistema tiene en cuanto a la conservación local de la biodiversidad, ya que pueden darse múltiples combinaciones entre los elementos, entre el sistema y elementos específicos, entre ellos y la flora y fauna silvestre remanente; esto tiene consecuencias sobre el movimiento de los organismos y sobre los procesos de transferencia de materia y energía entre unos y otros sectores del paisaje (DE MIGUEL, 1999). Para una configuración espacial cualquiera, la diversidad de un sector dependerá tanto de sus características intrínsecas (precipitación, litología, relaciones interespecíficas, etc.) como de las relaciones de dependencia que mantenga con otros sectores diferentes (DE MIGUEL, 1999), de allí la importancia de las cercas vivas en el mantenimiento de la diversidad local, de allí el valor de su conservación como elementos conectores de flujos energéticos.

La fragmentación del paisaje tropical, debida al proceso de transformación agrícola, se destaca por la presencia de grandes extensiones de potreros donde se observan ocasionalmente fragmentos remanentes de la vegetación natural, de distinta forma y tamaño, también árboles dispersos que actúan como sombrío y cercas vivas, que son el resultado de una práctica de explotación ancestral que aún no ha desaparecido (GUEVARA et al., 2005; BEJARANO-CASTILLO y GUEVARA, 2008). Es claro que los atributos de los árboles se pueden evidenciar y relacionar con la asociación faunística que posean muchas especies de aves y murciélagos, por ejemplo, así como con la producción de hojas, flores y frutos (GALINDO-GONZÁLEZ et al., 2000).

En este tipo de arreglo, las cercas vivas, en paisajes degradados y carentes de significativo arbolado, se convierten en invaluables bancos de semillas, que son sin duda los encargados de iniciar la regeneración natural de un área dada (GUEVARA et al., 2005). Por lo tanto, su valor no solo es presente sino que es activo y futuro. Existe una creciente tendencia a reconocer la necesidad de evaluar la capacidad de los agroecosistemas respecto de la conservación, en especial para aquellas áreas en donde la vegetación natural ha sido convertida en campos de producción agrícola o en donde el paisaje ha sido fuertemente fragmentado (HARVEY et al., 2006; GARBACH et al., 2010).

Las cercas vivas tienen una importante función en el medio ambiente, ya que ayudan a prevenir el movimiento del suelo ocasionado por las corrientes de agua, pueden influir en el ciclo de los nutrientes y el agua, además de ser 
reservorios para muchas especies de flora y fauna silvestre (CERVANTESCORNIHS et al., 2009).

GARBACH et al. (2010) consideran que las cercas vivas proveen un complejo vertical de árboles dentro de un sistema de pasturas simples, que puede proveer recursos para la fauna silvestre y conectividad entre parches de remanentes áreas forestales y boscosas primarias o secundarias. Se pueden considerar incorporadas a los sistemas productivos de fincas y son una barrera para el movimiento del ganado, proveen madera, plántulas para nuevas cercas y frutos aprovechables (HARVEY et al., 2005; LEÓN y HARVEY, 2006). No obstante, el manejo de las cercas vivas depende de manera particular de los intereses de sus propietarios, por lo tanto sus constitución estará dada en este sentido y decir que en todos los casos son elementos funcionales en conservación estará condicionado a la variedad y calidad de los elementos arbóreos que ella posea (GARBACH et al., 2010).

PAGIOLA et al. (2005) señalan que la importancia de las cercas vivas ha sido reconocida internacionalmente y que ha sido objeto de una amplia promoción en globales programas de conservación del Banco Mundial, con el fin de promover la conservación en áreas de producción agrícola. Sin embargo, los resultados de la adopción de las cercas vivas no han sido los esperados, se requieren mayor conocimiento, es necesario precisar los incrementos obtenidos en la conservación de la biodiversidad para determinar si se han logrado los efectos ecológicos deseables y poder así aumentar el establecimiento y uso de las cercas vivas con tal propósito.

No obstante, existe claridad en que las cercas multiestratales pueden tener un alto valor de conservación, pero menor a la que se puede hallar en parches de bosques con alto potencial de biodiversidad (BOCK et al., 2007; GARBACH et al., 2010). Igualmente se determina que los parches y las cercas vivas conectadas facilitan la movilización de diversos elementos faunísticos, en especial avifauna, dentro de un paisaje dominado por una matriz agrícola, aunque no exista en general calidad de hábitat permanente para cada unidad 0 submuestra del paisaje (GARBACH et al., 2010).

Las cercas vivas conformadas por mutiestratos soportan una mayor capacidad de forrajeo y de actividades de reproducción para la avifauna en comparación con las cercas vivas simples o recién establecidas, esto no sugiere que las cercas vivas puedan reemplazar hábitat primarios, pero si muestra que las cercas vivas multiestratales y las de mayor altura pueden ser de gran importancia en la provisión de recursos que requieren poblaciones de avifauna, además que pueden ser vital punto de apoyo en la migraciones (GARBACH et al., 2010). 
Como indicador, la ausencia o infrecuente presencia de algunas especies de aves en las cercas vivas y que se observan comúnmente en los parches y en los complejos agroforestales se asocia al aislamiento producido por grandes áreas de pasturas dominantes, que pueden ocasionar limitación en el movimiento de ciertas especies (WOOD y SAMWAYS, 1991). La ausencia de ciertas especies en áreas predominantes de pasturas sugieren que la configuración de parches de hábitat remanente determinan la distribución local, además que se tiene una pobre conectividad para estas especies (RICKETS et al., 2001).

En algunos casos la oferta de las cerca vivas puede no ser suficiente o puede darse un uso de plantas no preferidas como alternativa de alimentación, impidiendo de esta forma el establecimiento de las especies objeto de este forrajeo, situación similar, para parches de vegetación ha sido descrita por SUÁREZ-DUQUE (2008)

Según IBRAHIM et al. (2007) los enfoques clásicos de conservación, los cuales han estado dirigidos a preservar hábitat prístinos dentro de parques nacionales $\mathrm{u}$ otras categorías de áreas naturales protegidas, si bien son necesarios, no han resultado suficientes frente a las crecientes presiones para la conversión de hábitat naturales en tierras de producción. En este contexto, las tecnologías silvopastoriles ofrecen una alternativa ambientalmente amigable frente a los sistemas convencionales de producción ganadera.

IBRAHIM et al. (2007) sustentan que los sistemas silvopastoriles, entre los que se encuentran los árboles en las pasturas, los bancos forrajeros y las cercas vivas (PEZO y IBRAHIM, 2000) permiten mejorar la productividad de las pasturas y del ganado (HOLMANN et al., 1992; BETANCOURT et al., 2003), diversificar la producción (CURRENT et al., 1995) y generar incrementos en los ingresos (DAGANG y NAIR, 2003). Asimismo, los sistemas silvopastoriles son capaces de fijar cantidades importantes de carbono en el suelo y la biomasa aérea (IBRAHIM et al., 2007); proveer hábitat para biodiversidad (SAÉNZ et al., 2007) y conectividad al paisaje (HARVEY et al., 2005), y disminuir la escorrentía superficial (RÍOS, 2006). No obstante, como lo aseveran DAGANG y NAIR, (2003) pese a sus favorabilidades poseen bajos niveles de adopción. Según IBRAHIM et al. (2007), esto se asocia económicamente a los elevados costos iníciales para su establecimiento, los costos de oportunidad asociados con la demora en el tiempo hasta que los sistemas se vuelven productivos y la rentabilidad limitada que puedan llegar a presentar la mayoría de los mismos. Por otro lado, los beneficios ambientales de la incorporación de sistemas silvopastoriles, tales como captura de carbono o conservación de la biodiversidad, son poco conocidos por los productores.

\section{Fauna silvestre asociada}


La sobrevivencia de la fauna silvestre en áreas de uso agrícola y en hábitat de fragmentos depende de su habilidad para moverse dentro de estas áreas para acceder a los recursos necesarios, mantener su diversidad genética y asegurar su capacidad poblacional reproductiva (BUZA y THRALL, 2000), sin embargo, pequeñas poblaciones que se mueven entre fragmentos no logran acceder a los recursos necesarios y pueden tender a la extinción local (ANDRÉN, 2004).

Las cercas vivas son muy utilizadas por aves migratorias y residentes, se ha observado que a mayor densidad de cercas vivas en el paisaje, a distintas escalas espaciales, se incrementa la riqueza y la abundancia de aves (ZURIA y GATES, 2006). GARBACH et al. (2010) muestran que las cercas vivas en Costa Rica son importantes elementos para la avifauna del paisaje agrícola dominado por áreas de pasturas y que este valor está relacionado directamente con su estructura, además que la abundancia y riqueza de especies de aves incrementa con la complejidad de la estructura, siendo mayor entre mayor sea el número de estratos de que se disponga. Resultados similares se han reportado para corredores de vegetación de bosque lluvioso en Australia (LAURANCE y LAURANCE, 1999) y en corredores de vegetación riparia de zonas de agricultura (HILTY y MERENLENDER, 2004).

Como lo señalan KEYSER et al. (1998), la degradación de hábitats, la fragmentación y el aislamiento de bosques disminuyen las poblaciones de aves y afectan de manera distinta los diferentes gremios. Las rapaces de interior de bosque, las insectívoras terrestres y las frugívoras grandes son altamente propensas a la extinción local, mientras que las nectarívoras, granívoras, frugívoras pequeñas e insectívoras aéreas son más flexibles a los cambios en este tipo de paisaje (NARANJO y CHACÓN DE ULLOA, 1997; RENJIFO, 1999; WILSON et al., 1999; CROOKS et al., 2001).

Atendiendo lo conceptuado por KARR (1982) y STOUFFER y BIERREGAARD (1995) las aves de gran tamaño o de interior del bosque poseen reducida capacidad para movilizarse entre áreas abiertas, mientras que las especies de borde caracterizadas por su alta movilidad son más resistentes a ésta situación de fragmentación. La variación espacial y temporal de recursos, como frutos 0 néctar, hacen que especies de aves, que dependen de estos, sean altamente susceptibles a los cambios drásticos en la matriz del paisaje (LOISELLE y BLAKE, 1992; SEKERCIOĞLU et al., 2002; ENRÍQUEZ et al., 2009).

ENRÍQUEZ et al. (2009) en un estudio en una zona de agroecosistemas en Costa Rica, hallaron que el gremio de las insectívoras dominó la composición de la comunidad de aves, lo cual ha sido un patrón general en varios estudios realizados en otros agroecosistemas (ESTRADA et al., 1997; CÁRDENAS, 2003; CÁRDENAS et al., 2003; LANG et al., 2003; MENACHO y SÁENZ, 
2004). El gremio de las omnívoras, fue abundante, se considera versátil en el uso de diferentes recursos y puede acoplarse muy bien a los ambientes antrópicos, como en el caso de Turdus grayi o Crotophaga sulcirostris, las cuales son especies extremadamente abundantes en esta región (STILES y SKUTCH, 2003).

Para Costa Rica, en programas silvopastoriles, se han registrado especies de interés para la conservación de avifauna: pájaro campana (Procnias tricarunculata), buco barbón (Malacoptila panamensis), pinzón aceitunado (Arremonops rufivirgatus), pinzón piquinaranja (Arremon aurantiirostris), soterrey rufo y blanco (Thryothorus rufalbus), mosquero real (Onychorhynchus coronatus), gavilán blanco (Leucopternis albicollis); mariposas (Panthiades bathildis, Dynamine milita, Neographium epidaus). Todas esta especies son sensibles a la reducción de su hábitat natural, especialmente de la cobertura arbórea, por lo que es probable que con la implementación de los sistemas silvopastoriles efectuada por los productores en sus fincas se haya contribuido a la conservación de estas especies dentro del paisaje agropecuario (IBRAHIM et al., 2007).

SÁENZ et al. (2007) hallaron una alta correlación entre la riqueza de especies de aves y la riqueza y cobertura de la vegetación para un estudio comparativo entre Nicaragua, Costa Rica y Colombia. Al comprar la similitud de especies de aves entre Costa Rica y Colombia, encontraron que existía diferencia significativa, siendo la variable que crea la diferencia la mayor presencia de cercas vivas y árboles en potreros, más usuales en Costa Rica que en la zona de Colombia donde se llevó a cabo el estudio; enfatizan que este tipo de sistemas agrosilvopastoriles son muy utilizados por las aves y que en paisajes agrícolas muy tecnificados las aves tienden a desaparecer

Como registro notorio y puntual de la importancia de las cercas vivas en la conformación de una comunidad y sus relaciones, se tiene, según IDROBO y CORTÉS-HERRERA (2006) que en cercas vivas de un potrero ubicado en bosque seco premontano (bs-PM) de Colombia, conformado por guadua (Guadua angustifolia), dividivi (Caesalpinia pinnata) y nacedero o yátago (Trichanthera gigantea) un grupo de colibríes (Amazilia castaneiventris) (Trochilidae), además de libar las flores de un árbol de nacedero (Trichanthera gigantea), forrajearon y consumieron jejenes (Diptera: Simuliidae) que revoloteaban sobre el lomo de un caballo, igualmente se registró una posible relación de mutualismo facultativo entre el caballo y los colibríes; se les observó incluso alimentarse de los insectos mencionados que estaban posados sobre el animal, lo que muestra lo importante de las relaciones ecológicas entre esta especie de aves, las cercas y la fauna doméstica asociada. 
También se relaciona la importancia del nacedero (Trichanthera gigantea), común en cercas vivas y remanentes boscosos de los Andes colombianos, como especie que provee alimento y la más visitada por el colibrí endémico quincha de Soatá (Amazilia castaneiventris), pero que también posee relevante importancia para otras especies colibríes como: el picaflor (Chlorostilbon poortmani) (CHAVES-PORTILLA y CORTÉS-HERRERA, 2006), ampliándose así la importancia de las cercas vivas en esta zona.

En aves asociadas a zonas abiertas y cercas vivas, en Ecuador se ha registrado que tres especies del género Tangara (Thraupidae) ( $T$. heinei, $T$. parzudakii y $T$. nigroviridis) anidan y mantienen relaciones con este sistema, usando principalmente las siguientes especies arbóreas: Sapium sp. (Euphorbiaceae), Inga sp. (Fabaceae) y Saurauia sp.( Actinidiaceae). Además se localizan Lepidocolaptes lachrymiger (Furnariidae), Contopus fumigatus (tyrannidae), Thraupis palmarum (Thraupidae) y Pipraeidea melanonota (Thraupidae) (ARCOS-TORRES y SOLANO-UGALDE, 2007).

SANDOVAL (2009) informa que la codorniz de monte Colinus leucopogon, especie común en el bosque seco y el valle Central de Costa Rica, por debajo de $1.600 \mathrm{~m}$, habita potreros, áreas de crecimiento y cafetales, y que se trata de una especie adaptada a sistemas silvopastoriles y de hecho importante en la cadena de flujos de este sistema ambientales antropizados. Por su parte, TREJO et al. (2008) indican que para Venezuela la perdiz encrestada (Colinus cristatus) que se distribuye desde Panamá al este de Colombia, Venezuela y norte de Brasil, en las Antillas Menores cerca del continente, es una especie altamente apreciada por cazadores deportivos en toda su área de distribución. En Venezuela, se ubica en el tercer lugar en el número de reportes realizados por cazadores deportivos (BABARRO, 1998) y al igual que sus congéneres es habitante de zonas antropizadas, a las cuales se adapta bien.

Otra especie relacionada, la perdiz del norte (Colinus virginianus), presente en Norteamérica, que ha sido ampliamente estudiada (GUTHERY, 2000) y manejada cinegéticamente, provee por actividades relacionadas con su cacería deportiva y por turismo ecológico, la base de una industria que genera miles de dólares y empleo para muchas personas. En este caso, haciendo referencia a las perdices se tiene un valor de aprovechamiento que los paisajes agrosilvopastoriles pueden aportar y que no son despreciables, al tiempo que permiten la conservación de áreas de importancia.

No obstante, en sentido estricto de posible usos cinegéticos muchas especies de aves residentes de parches y cercas vivas podrían ser tenidas en cuenta para un uso sostenible y rentable, por ejemplo ESTELA et al. (2005) registran en cercas vivas la presencia de la tortolita escamada (Columbina squammata) para el Caribe colombiano. 
Se sabe que en las últimas décadas muchos depredadores de aves han expandido su distribución hasta ambientes agrícolas, urbanos y semiurbanos, ocasionando incremento en la depredación de nidos y consecuentemente ocasionando el decremento poblacional de algunas especies de aves (JOKIMÄKI y HUHTA, 2000). Las causas de esta expansión de depredadores a zonas antropizadas se fundamentan en la alta disponibilidad de alimento que estos ambientes ofrecen lo que genera la atracción de ciertos depredadores. Se sabe también que los hábitats lineales (como cercas vivas) que bordean las zonas agrícolas pueden funcionar como trampas ecológicas, tienen atributos específicos que atraen a las aves que anidan, pero al mismo tiempo atraen a sus depredadores, observándose altos índices de depredación de nidos (CERVANTES-CORNIHS et al., 2009).

La estructura de las cercas vivas, su densidad y su arreglo espacial en el paisaje, pueden afectar los patrones de depredación de nidos (ZURIA et al., 2007). No obstante, la pérdida poblacional de muchas especies avifauna en hábitats modificados, aún faltan estudios que analicen los efectos que tiene la depredación sobre las poblaciones de aves en relación a la expansión y la presencia humana (HASKELL et al., 2001; CERVANTES-CORNIHS et al., 2009).

En cuanto a mamíferos silvestres, MONGE y HILJE (2006) reportan la presencia de la ardilla (Sciurus variegatoides) especie de amplia distribución y habitante de zonas abiertas y alteradas desde el nivel del mar hasta los 2.500 $m$ de altura, incluyendo cercas vivas (BEST, 1995); se le considera una especie nociva, afecta diversos cultivos frutales como: banano (Musa sp.), papaya (Carica papaya), coco (Cocus nucifera), aguacate (Persea americana), mango (Mangifera indica), chayote (Sechium edule), y especies forestales como ceiba (Bombacopsis quinata) e incluso eucalipto (Eucalyptus spp.) (HILJE y MONGE, 1988). Igualmente consume almendro (Terminalia catappa), guayaba (Psidium guajava), guácimo (Guazuma ulmifolia), palma coroza (Attalea butyracea), jobo (Spondias mombin), camajón (Sterculia apetala) (JANZEN, 1972; GLANZ; 1984; MONGE y HILJE, 2006), especie arbóreas comunes en cercas vivas.

Igualmente, en el caso de insectos, existen registros de la cercas vivas como refugio y lugar de colonización de algunas especies de este grupo, como es el caso de Brevipalpus phoenicis (Acari: Tenuipalpidae), que trasmite la lepra viral de los cítricos, ocasionando pérdidas importantes en los naranjales en Brasil (MAIA y OLIVEIRA, 2004); esta enfermedad se considera una de la más graves para los cultivos de naranja dulce en Brasil (CHIAVEGATO et al., 1982; OLIVEIRA 1988) y se reconoce su presencia en países como Argentina, Bolivia, Paraguay, Uruguay y Venezuela (GARNSEY et al., 1989). Se relaciona además que determinadas especies vegetales no deberían estar asociadas a 
las cercas vivas que rodean o están en inmediaciones de los cultivos de cítricos, entre ellas: Achiote (Bixa orellana), hibisco (Hibiscus sp.) y malvavisco (Malvaviscus mollis) (ULIAN y OLIVEIRA, 2001).

IBRAHIM et al. (2007) muestran que al efectuar una comparación con la riqueza de especies presentes en un área protegida cercana a su área de estudio, los paisajes fragmentados pueden mantener hasta $67 \%$ de las especies de aves, $83 \%$ de las especies de mariposas y $77 \%$ de las especies de árboles presentes en un Parque Natural cercano (Santa Rosa), información que se apoya en estudio llevado a cabo por SÁENZ (2005). De lo anterior se desprende que es evidente que la diversidad arbórea presente en los sistemas silvopastoriles desempeñan un papel importante para la conservación de la fauna silvestre, aunque menor al de las áreas protegidas de bosque (IBRAHIM et al., 2007).

Respecto de las cercas vivas, el movimiento y dispersión de semillas llevado a cabo por frugívoros posee un alto valor ecológico, se destacan las aves, los murciélagos y en algunos casos los primates (PIERCE et al., 2001; TEWKSBURY et al., 2002)

\section{Conclusiones}

No es extraño que el aislamiento causado por la fragmentación de un ambiente limite el potencial de dispersión y colonización de plantas y animales, lo cual suprime sus necesidades fisiológicas, nutricionales y etológicas, que asociadas a problemas genéticos por ausencia de un adecuado flujo, pueda generar un escenario adecuado para procesos de extinción local o regional (PARDINI, 2004; WILSON, 2004); lo que muestra la importancia de la conectividad, la cual es factible en zonas agrícolas mediante el mantenimiento, promoción y extensión de la cercas vivas (ESTRADA et al., 2000; BENNETT, 2003).

Es evidente que los sistemas silvopastoriles, entre ellos las cercas vivas, cumplen una función de integralidad porque además de las especies vegetales existe un componente animal que permita la combinación de la actividad pecuaria con la plantación de árboles forrajeros, maderables, para leña y otros usos. Las cercas vivas, dadas estas características y su funcionalidad son en esencia, además de valiosos instrumentos de conservación faunística regional, un sistema silvopastoril de gran actualidad e importancia para el trópico.

Agradecimientos: A la Universidad de Sucre, Grupo de Investigación en Biodiversidad Tropical por su apoyo. A la Maestría en Ciencias Ambientales, SUE Caribe - Universidad de Sucre, este trabajo hace parte de la Tesis de Maestría en Ciencias Ambientales del autor. 


\section{Referencias}

ANDRÉN, H. 2004. Effects of habitat fragmentation on birds and mammals in landscapes with different proportions of suitable habitat: a review. Oikos 71: 355-366.

ARCOS-TORRES, A.; SOLANO-UGALDE, A. 2007. Notas sobre la anidación de tres especies del género Tangara (Thraupidae: Aves) en el noroccidente de Ecuador. Boletín SAO XVII (2):133-137.

BABARRO, R. 1998. Análisis de las temporadas de cacería deportiva en Venezuela a través de las temporadas 1987-88 y 1996-97. Informe técnico Servicio Autónomo Profauna, MARNR. Caracas, Venezuela.

BEER, J. 1987. Experiences with fence line fodder trees in Costa Rica and Nicaragua. Págs: 215-222. En: Beer, J.; Fassbender, H.W.; Hueveldop, J. (eds.). Advances in agroforestry research. Turrialba, CR, CATIE. Serie Técnica no. 147. Costa Rica.

BEER, J.; HARVEY, C.A.; IBRAHIM, M.; HARMAND, J.M.; SOMARRIBA, E.; JIMÉNEZ, F. 2003. Servicios ambientales de los sistemas agroforestales. Agroforestería en las Américas 10 (37):80-87.

BEJARANO-CASTILLO, M; S. GUEVARA, S. 2008. Algunos atributos de los árboles que atraen frugívoros a los potreros. Cuadernos de Biodiversidad 27:310.

BENNETT, A.F. 2003. Linkages in the landscape: The role of corridors and conncectivity in wildlife conservation. IUCN. Gland, Suiza.

BERNINGER, F.; SALAS, E. 2003. Biomass dynamics of Erythrina lanceolada as influenced by shoot-pruning intensity in Costa Rica.Agroforestry Systems 57: 19-29.

BEST, T.L. 1995. Sciurus variegatoides. Mammalian Species 500:1-6.

BETANCOURT, K.; IBRAHIM, M.; HARVEY, C.; VARGAS, B. 2003. Efecto de la cobertura arbórea sobre el comportamiento animal en fincas ganaderas de doble propósito en Matiguás, Matagalpa, Nicaragua. Agroforestería en las Américas 10 (39-40):47-51.

BOCK, C.E.; JONES, Z.; BOCK, J. 2007. Relationships between species richness, evenness, and abundance in a southwestern savanna. Ecology 88:1322-1327.

BOTERO, R.; RUSSO, O. 1998. Utilización de árboles y arbustos fijadores de nitrógeno en sistemas sostenibles de producción animal en suelos ácidos tropicales. Conferencia electrónica FAO sobre agro-forestería para la producción animal en Latinoamérica. Artículo 8. Disponible en: Utilización de 
arboles y arbustos fijadores de nitrógeno en sistemas sostenibles de producción animal en suelos ácidos tropicales. Consultado 15-10-2012.

BUZA, L.A.; THRALL, V. 2000. Genetic erosion, inbreeding and reduced fitness in fragmented populations of the endangered tetraploid pea Swainsona recta. Biological Conservation 93:177-86.

CÁRDENAS, G. 2003. Composición y estructura de la avifauna en diferentes sistemas de producción. En: Sánchez, M.D.; Rosales, M. (eds.). Agroforestería para la producción animal en América Latina. Memorias Segunda conferencia electrónica. Fao-Cipav.

CÁRDENAS, G.; HARVEY, C.A.; IBRAHIM, M.; FINEGAN, B. 2003. Diversidad y riqueza de aves en diferentes hábi-tats en un paisaje fragmentado en Cañas, Costa Rica. Agroforestería en las Américas 10:39-40.

CERVANTES-CORNIHS, E.; ZURIA, I.; CASTELLANOS, I. 2009. Depredación de nidos artificiales en cercas vivas de un sistema agro-urbano en Hidalgo, México. INCI 34 (11):777-783.

CHAVES-PORTILLA, G.; CORTÉS-HERRERA, O.J. 2006. Nueva localidad para la quincha de Soatá (Amazilia castaneiventris) en el municipio de San Gil, Santander, Colombia. Boletín SAO XVI (1):1-6.

CHIAVEGATO, L.G.; MISCHAN, M.M.; SILVA, M.A. 1982. Prejuízos e transmissibilidade de sintomas de leprose pelo ácaro Brevipalpus phoenicis (Geijskes, 1939) Sayed, 1946 (Acari, Tenuipalpidae) em citros. Científica 10: 265-271.

CORDERO, J.; BOSHIER D.H. 2003. Árboles de Centroamérica: Un manual para extensionistas. Oxford Forestry Institute, Catie. Oxford, UK - Costa Rica.

CROOKS, K.R.; SUAREZ, A.V.; BOLGER, D.T.; SOULÉ, M.E. 2001. Extinction and vcolonization of birds on habitats islands. Conservation Biology 15 (1):159172.

CURRENT, D.; LUTZ, E.; SCHERR, S.J. 1995. The costs and benefits of agroforestry to farmers." World Bank Research Observer 10 (2):151-180.

DAGANG, A.B.K.; NAIR, P.K. 2003. Silvopastoral research and adoption in Central America: Recent findings and recommendations for future directions. Agroforestry Systems 59:149-155.

DE MIGUEL, J.M. 1999. Naturaleza y configuración del paisaje agrosilvopastoril en la conservación de la diversidad biológica en España. Revista Chilena de Historia Natural 72:547-557.

DENIZ, A. 2003. Siembra directa, experiencia exitosa en el Estado de Colima. Forestal XXI 6 (3):23-24. 
ENRÍQUEZ, M.L.; SÁENZ, J.C.; IBRAHIM, M. 2009. Gremios de aves en agroecosistemas del Pacífico Central de Costa Rica y su importancia para la conservación. Ambientales 38:26-32.

ESQUIVEL, H.; IBRAHIM, M.; HARVEY, C.A.; VILLANUEVA, C.; BENJAMÍN, T.; SINCLAIR, F. 2003. Árboles dispersos en potreros de fincas ganaderas en un ecosistema seco de Costa Rica. RAFA 10:24-29

ESTELA, A.F.A.; JARAMILLO, B.J.G.; ALEJANDRO MEJÍA-TOBÓN, C.A. 2005. Ampliación de distribución de la tortolita escamada (Columbina squammata) en el Caribe colombiano. Boletín SAO XV (2):105-111.

ESTRADA, A.; CAMMARANO, P.L; COATES-ESTRADA, R. 2000. Bird species richness in vegetation fences and in strips of residual rain forest vegetation at Los Tuxtlas, Mexico. Biodiversity and Conservation 9:1399-1416.

ESTRADA, A.; COATES-ESTRADA, R. 2001. Bat species richness in live fences and in corridors of residual rain forest vegetation at Los Tuxtlas, Mexico. Ecography 24:94-102.

ESTRADA, A.; COATES-ESTRADA, R.; MERITT JR. D.A. 1997. Anthropogenic landscape changes and avian diversity at Los Tuxtlas, México. Biodiversity and Conservation 6 (1):19-43.

ESTRADA, A.; COATES-ESTRADA, R; MERITT JR, D; MONTIEL, S; CURIEL, D. 1993. Patterns of frugivore species richness and abundance in forest islands and in agricultural habitats at Los Tuxtlas, México. Vegetation 107/108:245257.

FEBLES, G.; RUIZ, T.E.; SIMÓN, L. 1995. Consideraciones acerca de la integración de los sistemas silvo-pastoriles a la ganadería tropical y subtropical. XXX Aniversario Instituto de Ciencia Animal. La Habana, Cuba:55-63.

GALINDO-GONZÁLEZ, J.; GUEVARA, S.; SOSA, V.J. 2000. Bat- and BirdGenerated Seed Rains at Isolated Trees in Pastures in a Tropical Rainforest. Conservation Biology 14:1693-1703.

GALLEGO, B. 2002. Estructura y composición de un paisaje fragmentado y su relación con especies arbóreas indicadoras en una zona de bosque muy húmedo tropical, de Costa Rica. Tesis de Maestría, CATIE, Turrialba, Costa Rica.

GARBACH, K.; MARTÍNEZ-SALINAS, A.; DECLERCK, F. 2010. La importancia del manejo: contribuciones de las cercas vivas para mantener la diversidad de aves en paisajes agrícolas. Mesoamericana Volumen 14 (3):49-64.

GARNSEY, S.M.; CHAGAS, C.M.; CHIAVEGATO, L.G. 1989. Leprosis and zonate chlorosis. Pág.43-44. En: WHITESIDE, J.O.; GARNSEY, S.M.; TIMMER, L.W. (eds.). Compendium of citrus diseases. St. Paul, A.P.S. Press. USA. 
GLANZ, W.E. 1984. Food and habitat use by two sympatric Sciurus species in Panama. J. Mamm. 65:342-347.

GUEVARA, S.; LABORDE, J.; SÁNCHEZ-RÍOS, G. 2005. Los árboles que la selva dejó atrás. Interciencia 10:595-601.

GUTHERY, F.S. 2000. On bobwhites. Moody WL, Natural History Series, № 27. Texas A\&M University Press. College Station. TX, EEUU.

GUTTERIDGE, R. 1990. Agronomic evaluation of tree and shurbs species in south east Quensland. Tropical Grassland 24:29-34.

HARVEY, C.A.; VILLANUEVA, V.; VILLACÍS, V.; CHACÓN, M.; MUÑOZ, D.; LÓPEZ, M.; IBRAHIM, M.; GÓMEZ, R.; TAYLOR, R.; MARTÍNEZ, J..; NAVAS, A.; SÁENZ, J.; SÁNCHEZ, D.; MEDINA, A.; VÍLCHEZ, S.; HERNÁNDEZ, B.; PÉREZ, A.; RUIZ, F.; LÓPEZ, F.; LANG, I.. 2005. Contribution of live fences to theecological integrity of agricultural landscapes in Central America. Agriculture, Ecosystems and Environment Journal 111:200-230.

HARVEY, C.A. 2001. The conservation of biodiversity in silvopastoral systems. International Symposium on silvopastoral systems and second Congress on agroforestry and livestock production in Latin America, silvopastoral systems for restoration of degraded tropical pasture ecosystems. San José, Costa Rica.

HARVEY, C.A.; HABER, W.A. 1999. Remanent trees and the conservation of biodiversity in Costa Rican pastures. Agrof. Systems 44:37-68.

HARVEY, C.A.; MEDINA, A.; SÁNCHEZ-MERLO, D.; VÍLCHEZ, S.; HERNÁNDEZ, B.; SÁENZ, J.; MAES, J.; CASANOVAS, F.; SINCLAIR, F.L. 2006. Patterns of animal diversity associated with different forms of tree cover retained in agricultural landscapes. Ecological Applications 16:1986-1999.

HARVEY, C.A.; VILLANUEVA, C.; IBRAHIM, M.; GÓMEZ, R.; LÓPEZ, M.; KUNTH S.; SINCLAIR, F. 2008. Productores, árboles y producción ganadera en paisajes de América Central: implicaciones para la conservación de la biodiversidad. Págs:197-224. En: HARVEY, C.; SAENZ, J. (eds.) Evaluación y conservación de biodiversidad en paisajes fragmentados de Mesoamérica. Santo Domingo de Heredia. Instituto Nacional de Biodiversidad (INBIO). Costa Rica.

HARVEY, C.A.; VILLANUEVA, C.; VILLACIS, J.; CHACÓN, M.; MUÑOZ, D.; LÓPEZ, M.; IBRAHIM, M.; GOMEZ, R.; TAYLOR, R.; MARTÍNEZ, J.; NAVAS, A.; SÁENZ, J.; SÁNCHEZ, D.; MEDINA, A.; VILCHEZ, S.; HERNÁNDEZ, B.; PÉREZ, A.; RUIZ, F.; LÓPEZ, F.; LANG, I.; KUNTH, S.; SINCLAIR, F.L. 2003. Contribución de las cercas vivas a la productividad e integridad ecológica de los paisajes agrícolas en América Central. Agroforestería en las Américas 10 (3940):30-39. 
HASKELL, D.G.; KNUPP, A.M.; SCHNEIDER, M.C. 2001. Nest predator abundance and urbanization. Págs:243-258. En: Marzluff, J.M.; Bowman, R.; Donnelly, R. (Eds.) Avian Ecology and Conservation in an Urbanizing World. Kluwer. Nueva York, EEUU.

HILJE, L.; MONGE, J. 1988. Lista preliminar y consideraciones generales acerca de los animales vertebrados plaga en Costa Rica. Manejo Integrado de Plagas 10:39-52.

HILTY, J.A.; MERENLENDER, A.M. 2004. Use of riparian corridors and vineyards by mammalian predators in Northern California. Conservation Biology 18:126-135.

HOLLMAN, F.; ROMERO, F.; MONTENEGRO, J.; CHANA, C.; OVIEDO, E.; BAÑOS, A. 1992. Rentabilildad de sistemas silvopastoriles con pequeños productores de leche en Costa Rica: primera aproximación. Turrialba 42 (1):7989.

IBRAHIM, M.; CHACÓN, M.; CUARTAS.; NARANJO, J.; PONCE, G.; VEGA, P.; CASASOLA, F.; ROJAS, J. 2007. Almacenamiento de carbono en el suelo y la biomasa aérea en sistemas de usos de la tierra en paisajes ganaderos de Colombia, Costa Rica y Nicaragua. Agroforestería en las Américas 45:27-36.

IBRAHIM, M.; MORA-DELGADO, J.; ROASALES, M. 2006. Conferencia electrónica: Potencia-lidades de los sistemas silvopastoriles para la generación de servicios ambientales (EAD-PFI-ECONF-L). En: www.flacsoandes.org/biblio/catalog/resGet.php?resld=51746. . Consultado: 0403-2013.

IBRAHIM, M.; T'MANNETJE, L.; OSPINA, S. 2003. Prospect and problems in the utilization of tropical herbaceous and woody leguminous forages. VI International Symposium on the nutrition of herbivores. Proceedings of an International Symposium held in Mérida, México:35-55.

IDROBO, C.J.; CORTÉS-HERRERA, J.O. 2006. Colibríes cazando jejenes: el caso de dos especies de Amazilia en los Andes colombianos. Boletín SAO XVI (2):40-45.

JANZEN, D. 1972. Escape in space by Sterculia apetala seeds from the bug Dysdercus fasciatus in a Costa Rican deciduous forest. Ecology 53:350-361.

JOKIMÄKI, J.; HUHTA, E. 2000. Artificial nest predation and abundance of birds along an urban gradient. Condor 102:838-847.

KAIMOWITZ, D. 2001. Will livestock intensification help save Latin Americas's tropical forest?. En: Angelsen, A.; Kaimowitz, D. (eds.). Agricultural technologies and tropical deforestation. Walling-Ford, UK.

KAIMOWITZ, D.; ANGELSEN, A. 2008. Will livestock intensification help save Latin America's forests? J. Sustain Forestry 27:6-24. 
KARR, J.R. 1982. Avian extinction on Barro Colorado Island, Panamá: a reassessment. American Naturalist 119 (2):220-239.

KEYSER, A.J.; HILL, G.E.; SOEHREN, E.C. 1998. Effects of forest fragment size, nest density and proximity to edge on the risk of predation to groundnesting passerine birds. Conservation Biology 12 (5):986-994.

KU, J. C.; RAMÍREZ, L.; JIMÉNEZ, G.; ALAYÓN, J.; RAMÍREZ, L. 1998. Árboles y arbustos para la producción animal en el trópico mexicano. Conferencia electrónica FAO sobre agro-forestería para la producción animal en Latinoamérica. Artículo $10 . \quad$ Disponible en: http://www.fao.org/ag/aga/agap/FRG/AGROFOR1/ku10.htm. Consultado: 1112-2012.

LAGEMANN, J.; HEUVELDOP, J. 1983. Characterization and evaluation of agroforestry systems: the case of Acosta-Puriscal, Costa Rica. Agroforestry Systems 1:101-115.

LANG, I.; GORMLEY, L.H.R.; CELIA A. HARVEY, C.A.; SINCLAIR, F.L. 2003. Composición de la comunidad de aves en cercas vivas de Río Frío, Costa Rica. Agroforestería en las Américas 10:39-40.

LAURANCE, S.G.; LAURANCE, W.F. 1999. Tropical wildlife corridors: use of linear rainforest remnants by arboreal mammals. Biological Conservation 91:231-239.

LEÓN, M.C.; HARVEY, C.A. 2006. Live fences and landscape connectivity in a neotropical agricultural landscape. Agroforestry Systems 68:15-26.

LOISELLE, B.A.; BLAKE, J.G. 1992. Population variation in a tropical bird community: implications for conservation. BioScience 42 (11):838-845.

LOZANO, O.R. 1962. Postes vivos para cercos. Tesis Mag.Sc. Turrialba,CR, CATIE/IICA. Manual de métodos de campo para el monitoreo de aves terrestres. United States Department of Agriculture Forest Service. Pacific Southwest Research Station General Technical Report PSW-GTR-159-Web. Albany, California.

MAIA, M.A.O; OLIVEIRA, A.L.C. 2004. Capacidade de Colonização de Brevipalpus phoenicis (Geijskes) (Acari: Tenuipalpidae) Em Cercas-Vivas, Quebra-Ventos e Plantas Invasoras Neotropical Entomology 33 (5):625-629.

MARTÍNEZ, J. 2004. Conocimiento local de productores ganaderos sobre cobertura arbórea en la parte baja de la cuenca del río Bulbul en Matiguas, Nicaragua. Tesis de Maestría, CATIE, Turrialba, Costa Rica.

MEDINA, A.; HARVEY, C.A.; SÁNCHEZ, V.; VILCHEZ, S.; HERNÁNDEZ, B. 2004. Diversidad y composición de chiropteros en un paisaje fragmentado de bosque seco en Rivas, Nicaragua. Managua, Nicaragua. Encuentros 68:24-43. 
MENACHO, R.; SÁENZ, J. 2004. Informe de Biodiversidad de primer año. Proyecto Enfoques Silvopastoriles Integrados para el Manejo de Ecosistemas. Una. Costa Rica.

MEYERS, N.; MITTERMEIER, R.A.; MITTERMEIER, C.G.; DA FONSECA, G.A.; KENT, J. 2000. Biodiversity hotspots for conservation priorities. Nature 403:853-858.

MONGE, J.; HILJE, L. 2006. Hábitos alimenticios de la ardilla Sciurus variegatoides (Rodentia: Sciuridae) en la Península de Nicoya, Costa Rica. Rev. Biol. Trop. 54 (2):681-686.

MURGUEITIO, E.; CALLE, Z. 1998. Diversidad biológica en sistemas de ganadería bovina en Colombia. Conferencia electrónica FAO sobre agroforestería para la producción animal en Latinoamérica. Artículo 3. Disponible en: $\quad$ http://www.fao.org/ag/aga/agap/frg/AGROFOR1/MURGUEI3.PDF. Consultado: 09-09-2012.

NARANJO, L.G.; CHACÓN DE ULLOA, P. 1997. Diversidad de insectos y aves insectívoras de sotobosque en hábitats perturbados de selva lluviosa tropical. Caldasia 19 (3):507-520.

OLIVEIRA, C.A.L. 1988. Ácaros: Fator de redução da produtividade das plantas cítricas. Pág.101-109. En: Donadio, L.C. (ed.). Produtividade do citros. FUNEP. Jaboticabal, Brasil.

PAGIOLA, S.; AGOSTINI, P.; GOBBI, J.; DE HAAN, C.; IBRAHIM, M.; MURGUEITIO, E.; RAMÍREZ, E.; ROSALES, M.; RUIZ, J.P. 2005. Paying for biodiversity conservation services: experience in Colombia, Costa Rica, and Nicaragua. Mountain Research and Development 25:206-211.

PALMA, J.M. 1998. Informe de resultados sobre el proyecto de Programa de Transferencia de Tecnología para la ganadería de doble propósito en el estado de Colima. Fundación PRODUCE. Colima, México.

PALMA, J.M. 2005. Los árboles en la ganadería del trópico seco. Avances en Investigación Agropecuaria 9 (1):1-11.

PALMA, J.M; FLORES, R. 1997. Aproximación al estudio de la vegetación arbórea del estado de Colima, México. X Reunión de Avances en Investigación Agropecuaria, Trópico '97. Barra de Navidad, Jalisco: 88-90.

PARDINI, R. 2004. Effects of forest fragmentation on small mammals in an Atlantic Forest landscape. Biodiversity and Conservation 13:2567-2586.

PEZO, D.; IBRAHIM, M. 2000. Sistemas Silvopastoriles. Módulo de Enseñanza Agroforestal. CATIE. Turrialba, Costa Rica. 
PIERCE, R.A. II; FARRAND, D.T.; KURTZ, W.B. 2001. Projecting the bird community response resulting from adoption of shelterbelt agroforestry pratices in Eastern Nebraska. Agroforestry Systems 53:333-350.

PITHER, R.; KELLMAN, M. 2002. Tree species in small tropical riparian forest fragments in Belize, Central America. Biodivers. Conserv. 11:1623-1636.

RENJIFO, L.M. 1999. Composition changes in a subandean avifauna after longterm forest fragmentation. Conservation Biology 13 (5):1124-1139.

RICKETS, T.H.; DAILY, G.C.; ERLICH, P.R.; FAY, J.P. 2001. Countryside biogeography of moths in a fragmented landscape: species richness in native and agricultural habitats. Conservation Biology 16:378-388.

RíOS, J. 2006. Comportamiento hidrológico de sistemas de producción ganadera convencional y silvopastoril en la zona de recarga hídrica de la subcuenca del Río Jabonal, cuenca del río Barranca, Costa Rica. Tesis Mag. Sc. CATIE. Turrialba, Costa Rica.

RUIZ, T.E.; FEBLES, G. 1999. Sistemas silvo-pastoriles. Conceptos y tecnologías desarrolladas en el Instituto de Ciencia Animal de Cuba. EDICA, La Habana, Cuba.

SÁENZ, J.C. 2005. Informe monitoreo de aves Esparza, Costa. Proyecto GEFCATIE. Turrialba, Costa Rica.

SÁENZ, J.C.; VILLATORO, F, IBRAHIM, M.; FAJARDO, D.; PÉREZ, M. 2007. Relación entre las comunidades de aves y la vegetación en agropaisajes dominados por la ganadería en Costa Rica, Nicaragua y Colombia. Agroforestería en las Américas 45:37-48.

SALAS, J. 1993. Árboles de Nicaragua. Servicio Forestal Nacional. HISPAMER. Managua, Nicaragua.

SÁNCHEZ-MERLOS, D.; HARVEY, C.A.; GRIJALVA,A.; MEDINA, A.; VÍLCHEZ, S.; HERNÁNDEZ; B. 2005. Diversidad, composición y estructura de la vegetación en un agropaisaje ganadero en Matiguás, Nicaragua. Rev. biol. trop $53(3-4): 387-414$.

SANDOVAL, L. 2009. Descripción del comportamiento de cuido parental en la codorniz de monte Colinus leucopogon. World Pheasant Association. International Journal of Galliformes Conservation 1:36-40

ŞEKERCIOḡLU, C.H.; EHRLICH, P.R.; DAILY, G.C.; AYGEN, D.; GOEHRING, D.; SANDÍ, R.F. 2002. Disappearance of insectivorous birds from tropical forest fragments. PNAS 99 (1):263-267.

SERRAO, E.A.S.; TOLEDO, J.M. 1990. The search for sustainability in Amazonian pastures. Anderson, A.B. (ed.).Columbia University Press. NY, EEUU. 
SHELTON, H.; LOWRY, J.; GUTTERIDGE, R.; BRAY, R.; WILSON, J. 1991. Sustaining productive pastures in the tropics. 7. Tree and shurb lehumes in improved pastures. Tropical Grassland 25:119-128.

SILVA-MATOS, D.M.; FONSECA, G.D.F.M.; SILVA-LIMA, L. 2005. Differences on post-fire regeneration of the pioneer trees Cecropia glazioui and Trema micrantha in a lowland Brazilian Atlantic Forest. Rev. Biol. Trop. 53:1-4.

SOMARRIBA, E. 1990. ¿Qué es agroforestería? El Chasqui 24:3-10.

SOMARRIBA, E. 1995. Guayaba en los potreros: establecimiento de cercas vivas y recuperación de pasturas degradadas. Agroforestería de las Américas 6:27-29.

STILES, G.; SKUTCH, A. 2003. Guía de las aves de Costa Rica. Tercera edición. Santo Domingo de Heredia: INBio. Costa Rica.

STOUFFER, P.C.; BIERREGAARD, R.O. JR. 1995. Use of Amazonian forest fragments by understory insectivorous birds. Ecology 76:2429-2445.

SUÁREZ-DUQUE, D. 2008. Formación de un corredor de hábitat de un bosque montano alto en un mosaico de páramo en el norte del Ecuador. Ecología Aplicada 7 (1-2):9-15.

TEWKSBURY, J.J.; LEVEY, D.J.; HADDAD, N.M.; SARGENT, S.; ORROCK, J.L.; WELDON, A.; DANIELSON, B.J.; BRINKERHOFF, J.; DAMSCHEN, E.I.; TOWSEND, P. 2002. Corridors affect plants, animals, and their interactions in fragmented landscapes. Proceedings of the National Academy of Sciences of the United States of America 99:12923-12926.

TOBAR-LÓPEZ, D.; IBRAHIM, M. 2008. Valor de los sistemas silvopastoriles para conservar la biodiversidad en fincas y paisajes ganaderos en América Central. Serie técnica. Informe técnico No. 373. CATIE, Costa rica.

TREJO, E.; MCNEIL, R.; MORALES, L.G.; LAU, P. 2008. Desplazamientos diarios, área de vivienda y utilización de los hábitat por perdices encrestadas (Colinus cristatus) marcadas con radios, en una sabana de Venezuela. Interciencia 33 (3):207-212.

ULIAN, L.F.; OLIVEIRA, C.A.L. 2001. Proteção integrada: O ácaro da leprose em diferentes cercas-vivas e quebraventos. Fundecitrus 105:12.

VILCHEZ, S.; HARVEY, C.A.; SÁNCHEZ, D.; MEDINA, A.; HERNÁNDEZ, B. 2004. Diversidad de aves en un paisaje fragmentado de bosque seco en Rivas, Nicaragua. Managua, Nicaragua. Encuentros 68:60-75.

WILSON, J.D.; MORRIS, A.J.; ARROYO, B.E.; CLARK, S.C.; BRADBURY, R.B. 1999. A review of the abundance and diversity of invertebrate and plant 
foods of granivorous birds in Northern Europe in relation to agricultural change. Agriculture, Ecosystems and Environment 75 (1-2):13-30:

WILSON, M.F. 2004. Loss and habitat connectivity hinders pair formation and juvenile dispersal of chucao tapaculos in Chilean rainforest. Condor 106:166171.

WOOD, P.A.; SAMWAYS, M.J. 1991. Landscape element pattern and continuity of butterfly fligth paths in a ecologically landscaped botanic garden, Natal, South Africa. Biological Conservation 58:149-166.

ZURIA, I.; GATES, J.E. 2006. Vegetated field margins in México: Their history, structure and function and management. Human Ecol. 34:53-77.

ZURIA, I.; GATES, J.E.; CASTELLANOS, I. 2007. Artificial nest predation in hedgerows and scrub forest in a human-dominated landscape of central Mexico. Acta Oecol. 31:158-167. 